\title{
Sales Supporting System using Mobile Agent Technology
}

\author{
Kiyotaka SEKI \\ Senior Researcher, Laboratory Head, Telecommunications Systems G., \\ Signalling and Telecommunications Technology Div. \\ Shoko SUZUKI \\ Assistant Senior Researcher, Passenger Information Systems G., \\ Transport Information Technology Div.
}

\begin{abstract}
We have developed a system for sales staff to access database systems which contain the information on customers and travel plans through mobile terminals, such as a notebook Personal Computer (PC), from their location. This system uses a Java-based mobile agent system. Although it takes a comparatively long time for the first retrieval because the agent has to move to the mobile terminal, it is allowable for practical use. After the first retrieval, the communication time is nearly equal to the time for data transmission. We also propose the Intranet configuration where the mobile terminal can access to the server from on-board Local Area Network (LAN).
\end{abstract}

Keywords : mobile agent, mobile telecommunications, Internet protocol, Java, Intranet

\section{Introduction}

The agent technology is becoming increasingly important in recent years. The mobile agent is a software agent focused on the mobility over the network and can move from one computer to another for execution. The mobile agent technology is thought to be one of the core technologies for distributed systems in the next generation, and a number of development tools are provided by various organizations and companies. Many of the tools are Java $^{\mathrm{TM}}$-based ${ }^{1) 2)}$, some of which provide not only the mobility function but also the communication function between agents and the function to reproduce its own schedule according to the environment to be visited.

As the wireless telecommunication technology and electronic device technology progress on the other hand, mobile computing which enables us to process information anytime and anywhere is now being realized. Examples of media which provide advanced wireless telecommunications are Personal Digital Cellular System (PDC), Personal Handy-phone System (PHS) which provides digital micro cellular phone services, Wireless LAN and International Mobile Telecommunication-2000 (IMT2000).

To realize advanced railway information processing systems, it is essential to consider communications and collaborations between mobile entities such as trains, crew, station staffs, trackside workers, and fixed entities such as dispatchers, host computers and so on. As the communication link can be established with the wireless technology described above, we consider that advanced systems can be constructed effectively by combining the agent technology and the mobile communication technology. Thus, it is possible to construct a more advantageous system than by traditional technologies where communication costs are not negligible or communication links are unstable, because it is necessary to set up communica- tion links when an agent moves or communicates with other agents. An example of these applications is a system which requires vast processing on servers. Another merit is that the destination computer doesn't need resources such as CPU and memory except for execution time and a hard disk drive to store programs. As a result, it is possible to simplify mobile terminals and omit its program maintenance work.

We have developed a system for sales staff to access database systems which contain the information on customers and travel plans through mobile terminals, such as a notebook PC, from their location. In this paper, we first describe the specification of this system. Then we introduce how to construct an Intranet for a railway company which enables the sales staff to make use of the mobile terminal on a train. This method makes possible for the on-board network to be treated seamlessly as a part of the Intranet of the railway company, and all the Internet applications as well as the mobile agent system can be used on-board. Finally, we describe the results of experiments.

\section{Specification}

In this section, we introduce the specification of this system.

\subsection{Configuration}

Figure 1 shows the configuration of the system. The sales staff who possesses a mobile terminal accesses the database system for information on customers and travel plans from his/her location, at tentative windows during busy seasons, or on a train. A database maintenance staff adds, updates or removes information on travel plans and sales staff. 


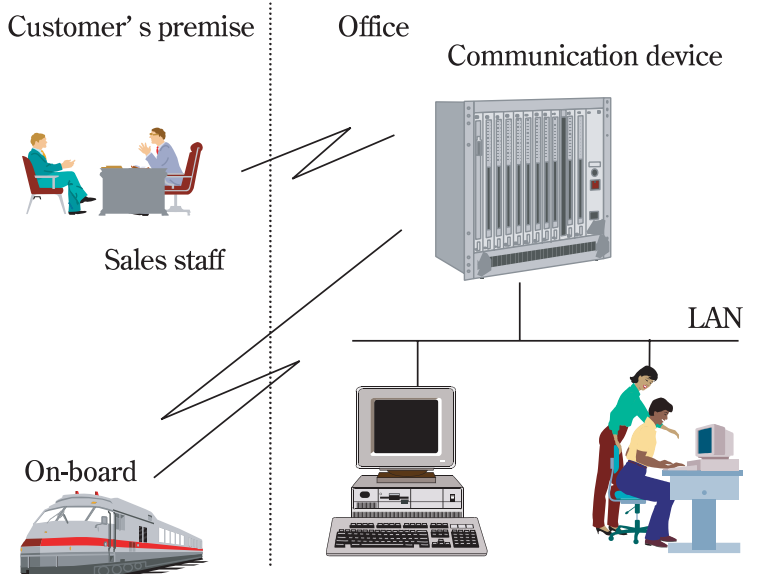

Database server Database maintenance staff

\subsection{Overview of processing}

Table 1 summarizes the implemented functions. This system is implemented with Aglets ${ }^{3)}$ as the mobile agent system. We use package tours as travel plans.

\subsubsection{Mobile information retrieval function}

Figure 2 illustrates an overview of the processing for the mobile information retrieval function.

(1) Authentication

When designated, a login agent is activated at a mobile terminal and displays a login menu for which the sales staff inputs a user ID and password.

The login agent is dispatched to an authentication server with input data and creates an authentication agent. The authentication agent accesses a sales staff database and notices the result of the authentication via communication between agents.

When the authentication is successful, the login agent creates an information retrieval agent, a customer database management agent, and a travel plan database man-

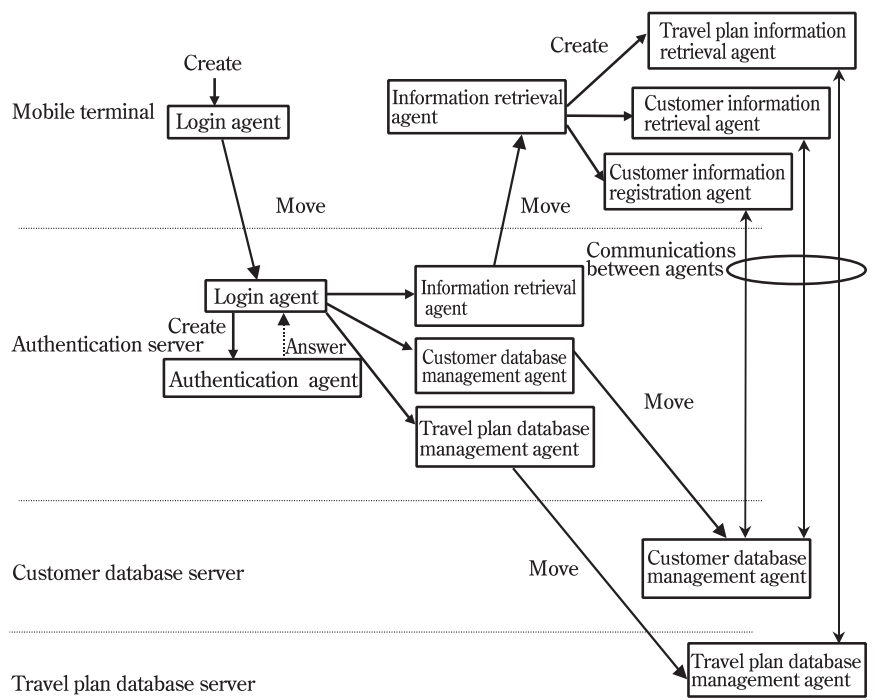

Fig. 2 Overview of the processing for the mobile information retrieval function

Table 1 Summary of functions

\begin{tabular}{|l|l|}
\hline Function name & Description \\
\hline \hline Mobile information retrieval & $\begin{array}{l}\text { Sales staff with mobile terminal retrieves } \\
\text { customer information and travel plan infor- } \\
\text { mation and makes reservation. }\end{array}$ \\
\hline System environment setting & $\begin{array}{l}\text { Database maintenance staff sets the opera- } \\
\text { tional environment of database servers and } \\
\text { registers, modifies or deletes the sales staff } \\
\text { information. }\end{array}$ \\
\hline Travel plan maintenance & $\begin{array}{l}\text { Database maintenance staff registers, } \\
\text { modifies or deletes travel plans and hotel } \\
\text { information. }\end{array}$ \\
\hline
\end{tabular}

agement agent and becomes extinct. The three created agents are dispatched to appropriate machines (mobile terminal, database servers ).

The information retrieval agent displays a menu from which the sales staff can select customer information retrieval, travel plan information retrieval or customer registration.

(2) Customer information retrieval

When selected, a customer information retrieval agent is created and requests input of retrieval conditions (customer's name, customer ID or customer's telephone number). The customer information retrieval agent then asks the customer database management agent about a list of the customers who satisfy the conditions via communications between agents and displays it. The customer database management agent creates SQL sentences and performs information retrieval.

When the sales staff selects a customer from the list, detailed information is displayed (Fig. 3). The staff can edit or delete the customer information including his/her name and address in the left half of this display. The right half shows the customer's history of purchase.

After that, the sales staff can select the travel plan information retrieval.

(3) Travel plan information retrieval and reservation When selected, a travel plan retrieval agent is cre-

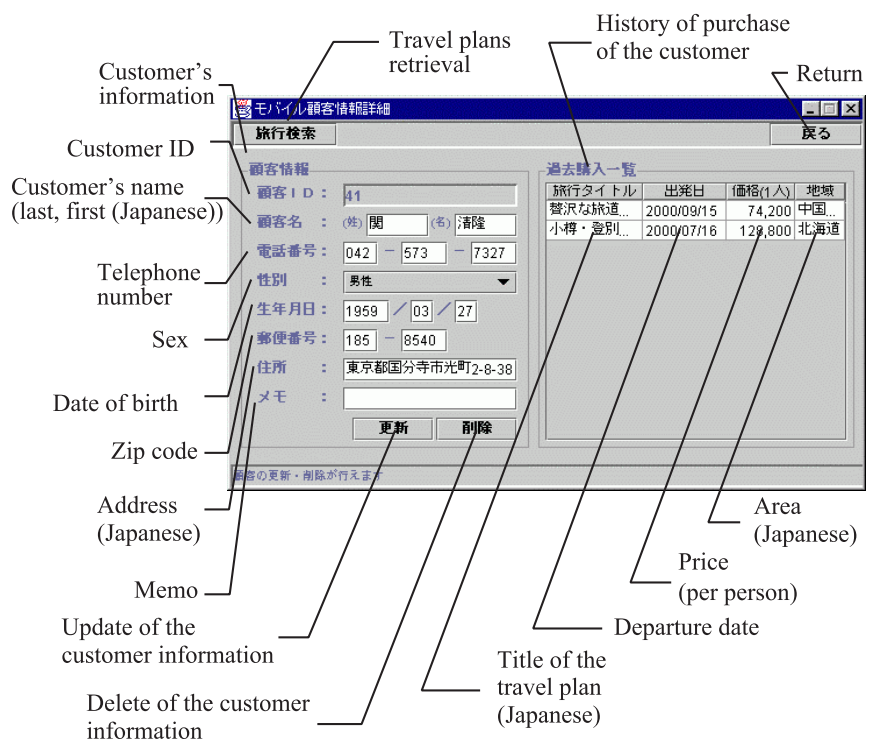

Fig. 3 Examples of the display of customer's detailed information 


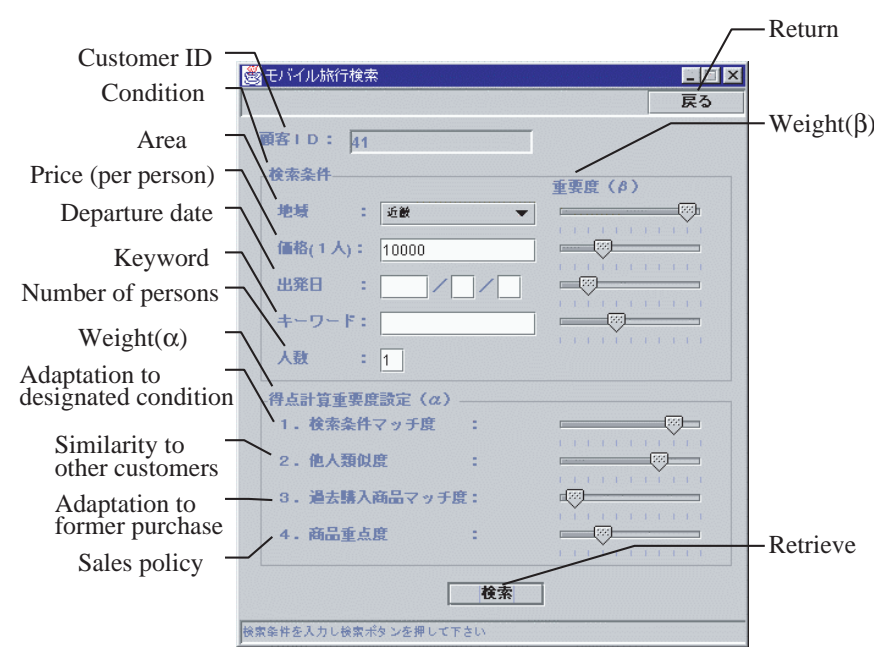

Fig. 4 Example of travel plan retrieval request

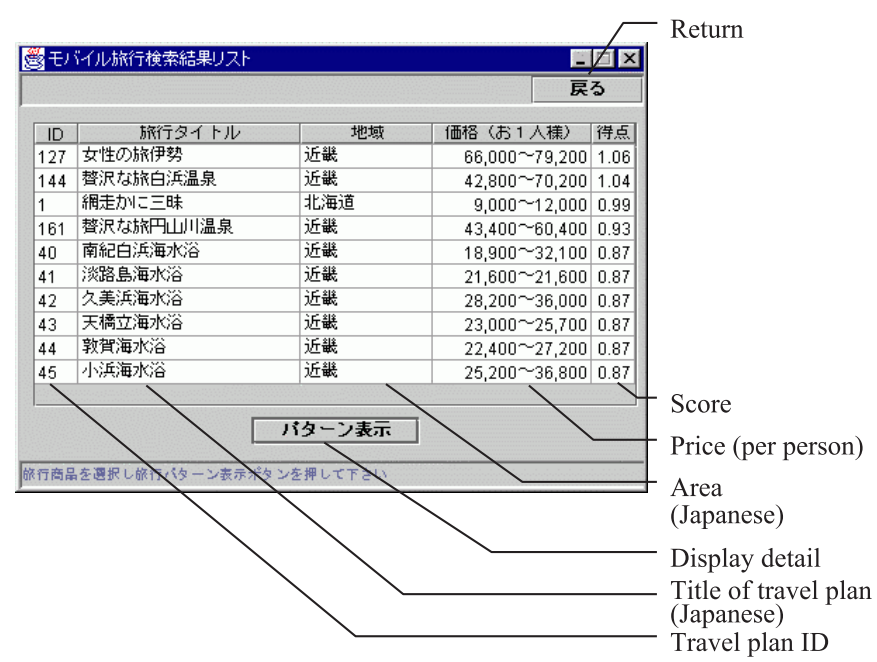

Fig. 5 Example of the result of travel plan retrieval

ated and requests input of retrieval conditions (Fig. 4). The travel plan retrieval agent then asks the travel plan database management agent about a list of the retrieved travel plans and displays it (Fig. 5). The travel plan database management agent calculates scores of the travel plans according to the designated conditions and answers the travel plans with high scores. Section 2.3 describes the retrieval conditions and how to assign the score to each travel plan.

When the sales staff selects a travel plan from the list, the detailed information of the travel plan is displayed. After the input of departure date, the reservation of the travel plan is completed.

(4) Customer registration

When selected, a customer registration agent is created and requests input of the customer's information (customer's name, customer's telephone number, etc. which is displayed on the left half of Fig. 3). The customer registration agent then asks the customer database management agent about the registration of the customer via communications between agents.

Merits of this configuration are as follows.

(a) As the agent for the process moves to the mobile ter- minal from the server at the execution time, it is not necessary to upgrade the program of any mobile terminal.

(b) There is no data or program at the mobile terminal except the login agent, so the possibility of leaking information is very low even if the mobile terminal is lost or stolen.

(c) The agent can access multiple database systems with only one communication protocol because the differences between specifications of database systems are absorbed by database management agents.

2.2.2 System environment setting and travel plan maintenance functions

Although these two functions are usually performed from terminals on the same local area network as database servers, they are implemented by using a mobile agent in order to match the mobile information retrieval function. The authentication process is the same as that of the mobile information retrieval function. In these functions, however, an agent corresponding to the selected process is created on the server instead of the terminal, because the communication capacity between the terminal and servers is very large.

In the system environment setting and travel plan maintenance functions, the processes listed in Table 2 and 3 are implemented.

\subsection{Scoring}

When travel plans are retrieved, the score is calculated for each travel plan, and travel plans are listed in the descending order of their scores. We adopt the following formula to calculate the score.

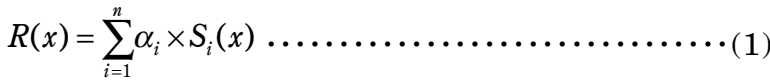

where $R(x)=$ Score of travel plan $x$

$S_{i}(x)=$ Score contributed by criterion $i$

$\alpha_{i}=$ Weight for criterion $i$ $n=$ Number of criteria

That is, we think that the score of a travel plan is given by the weighted sum of scores contributed by some criteria. In this trial manufacture, we adopt the following four criteria (i.e., $n=4)$.

Table 2 Processes provided by the system environment setting

\begin{tabular}{|l|l|}
\hline Name & Description \\
\hline \hline Servers environment setting & Set servers' name, port numbers, etc. \\
\hline Scoring function setting & $\begin{array}{l}\text { Set the default value of the scoring function } \\
\text { (see 2.3). }\end{array}$ \\
\hline Sales staff maintenance & $\begin{array}{l}\text { Register, update or delete sales staff } \\
\text { information. }\end{array}$ \\
\hline
\end{tabular}

Table 3 Processes provided by the travel plan maintenance function

\begin{tabular}{|l|l|}
\hline Name & Description \\
\hline \hline Travel plan maintenance & $\begin{array}{l}\text { Register, update or delete travel plan } \\
\text { information. }\end{array}$ \\
\hline Hotel maintenance & Register, update or delete hotel information. \\
\hline
\end{tabular}




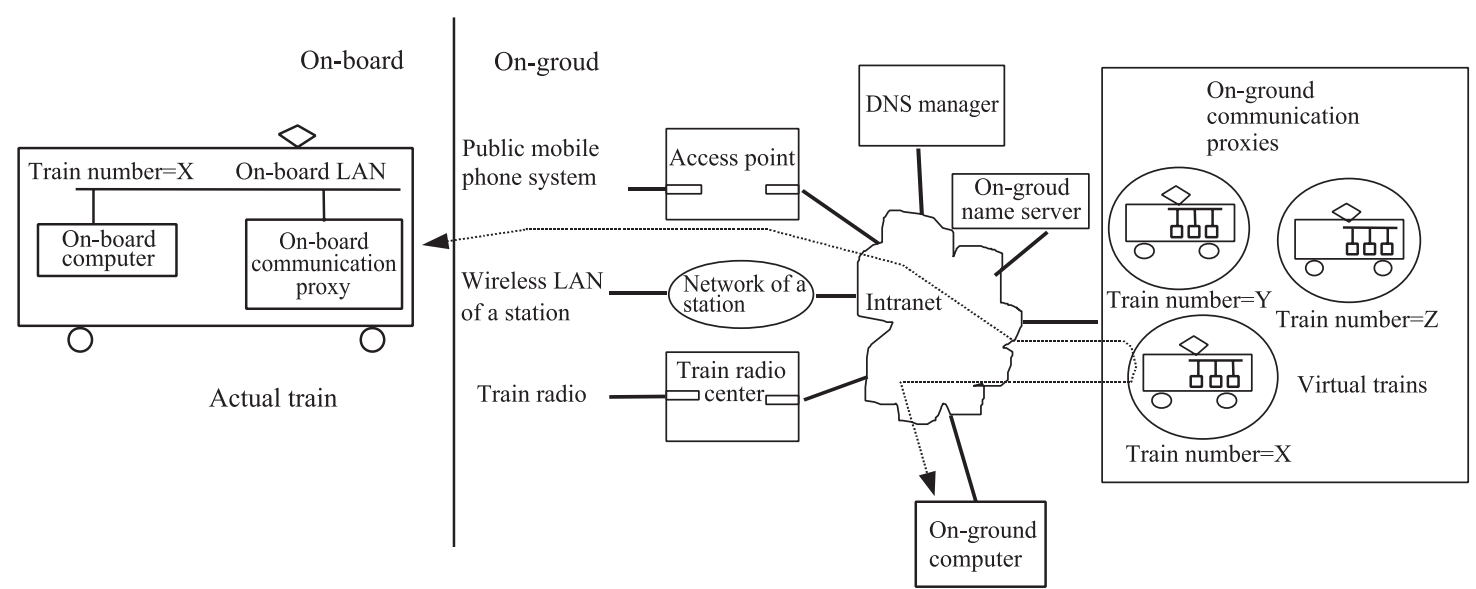

Fig. 6 The Intranet configuration for railways

(1) $S_{1}(x)$ :Adaptation to designated conditions

This criterion evaluates the conditions requested by a customer. $S_{1}(x)$ is calculated by eq. (2).

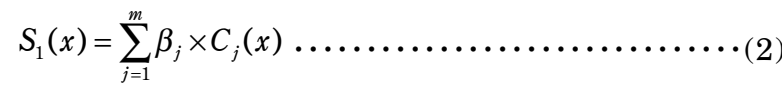

where $C_{j}(x)=$ Score contributed by condition $\mathrm{j}$

$\beta_{j}=$ Weight for condition $j$

$m=$ Number of conditions

In this trial manufacture, we adopt four conditions (i.e., $m=4$ ); area, price, departure date and keyword. The values of $\alpha$ s and $\beta$ s can be set by slide bars (see Fig. 6). (2) $S_{2}(x)$ :Similarity to other customers

A high score is given to the travel plans purchased by other customers who have the similar interest in the target customer. The similarity of interest is thought to be measured by how many travel plans of the same contents have been purchased by two customers.

$$
S_{3}(x)=\sum_{k=1}^{p} D_{k}(x)
$$

where $D_{k}(x)=$ Degree of the similarity of interest between the target customer and another customer $k$ who has purchased $x$.

$p=$ Number of customers who have purchased $x$ (3) $S_{3}(x)$ :Coincidence with the history of purchase

The customer's interest is extracted from his/her history of purchases. The travel plans which are thought to match his/her interest are given high scores.

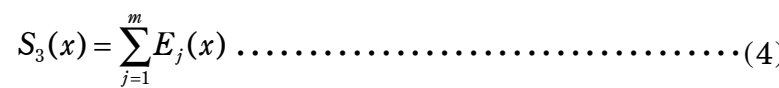

where $E_{j}(x)=$ Degree of the customer's interest in condition $\mathrm{j}$

$m=$ Number of conditions (same as in eq. (1))

(4) $S_{4}(x)$ :Promotion to travel plan

The database maintenance staff gives this score when he/she registers the travel plan according to the sales policy.

\section{Configuration of IP-based network in railways}

Mobile agents can move on the Internet or the Intranet whose basic protocol for data exchange is Internet Protocol (IP). Therefore, when the sales staff uses the mobile terminal at his/her location, it is necessary to have access to the network in a company by a public mobile phone installed to the mobile terminal. On the other hand, when the mobile terminal is used on a train, it can have access to the servers located on the ground only by connecting to on-board LAN using wireless LAN or Bluetooth technologies for example, if the on-board LAN is configured and recognized seamlessly as a part of the Intranet of a railway company. Of course, if this network is realized, not only the mobile agent system but also any Internet applications can be constructed including an on-board network. In this section, we describe the network structure for railways we propose.

\subsection{Network configuration}

The following items should be considered for the Intranet for railways including on-board LAN.

(a) Multiple wireless links such as the train radio system and public mobile phone system may connect between trains and the ground. The quality of links and communication costs of each wireless system vary, and usable locations are restricted. Therefore, the best wireless links should be selected by taking into consideration train locations and contents of transmission.

(b) Not the mobile terminal but the network itself is moving. Servers which are accessed from the ground may be connected to the on-board LAN. Therefore, an IP address of an on-board computer, which are used to identify each computer uniquely, should be invariant.

(c) Domain Name System (DNS) ${ }^{4) 5}$, which manages the correspondence between IP addresses and host names of computers, is operated in the Internet and Intranet. It is necessary to designate on-board computers by the host name including train numbers, for example, "onboard mail server of train number X." Therefore, the host name of the on-board computer changes as the train number of the train set on which the computer is installed changes. 


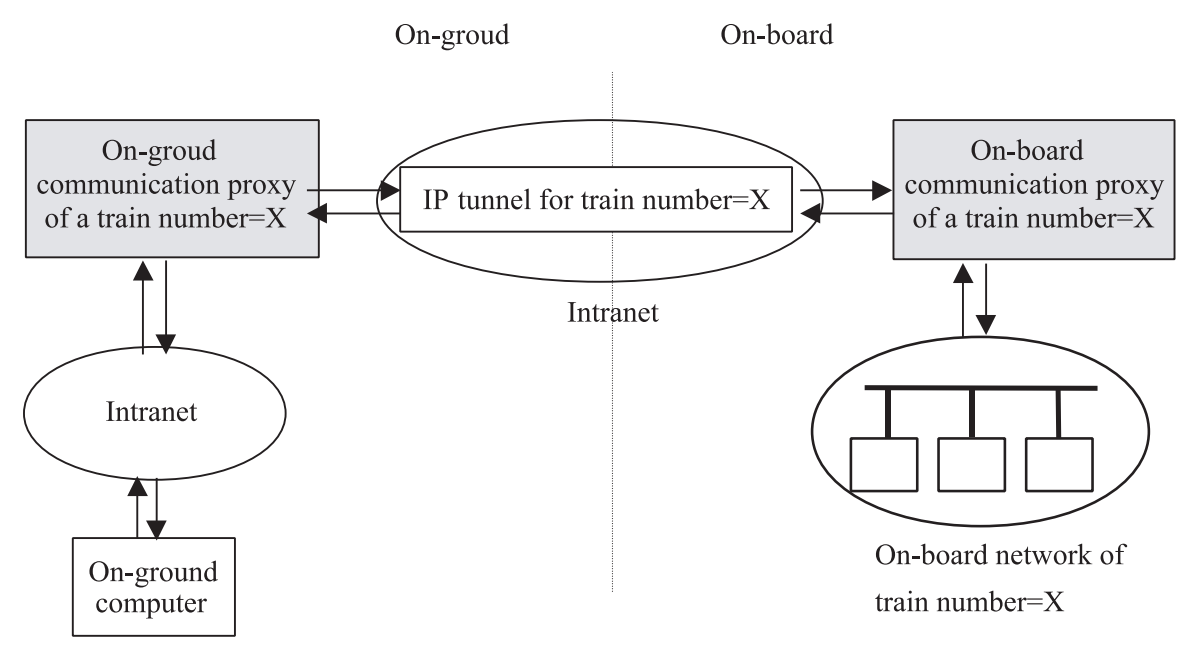

Fig. 7 Communication proxies and tunneling technique

Figure 6 shows the basic network configuration we propose to resolve the above-mentioned problems. Mobile $\mathrm{IP}^{6)}$ is a protocol applicable to mobile environments, but it is not realistic to use this protocol because communication traffic grows and all the computers connected to the on-board LAN have to implement this protocol. The proposed method enables computers connected to both onground and on-board networks to communicate with each other seamlessly without awareness of the connection status of wireless links. Communication proxies to exchange IP packets and a DNS manager to manage the correspondence between host names and IP addresses are operating on the networks in this proposal.

\subsection{Communication proxy}

There are on-ground and on-board communication servers, on which on-ground and on-board communication proxies operate, respectively. A pair of on-ground and on-board communication proxies exist for each train to construct and deconstruct IP tunnels according to the status of wireless links (Fig. 7). IP tunneling is a network technology to virtually realize the state where two computers located at separate places on the network are directly connected. The on-ground computer requests the on-ground communication proxy to relay a data packet to the on-board computer. Then, the on-ground communication proxy sends the packet to the on-board communication proxy through the IP tunnel, and the on-board communication proxy finally sends it to the on-board computer, that is, the destination of the packet. The data flow from an on-board computer to an on-ground computer is completely reverse. That is, all IP packets transferred between on-ground and on-board computers always go through these proxies. Therefore, the on-ground computer can communicate with on-board computers without being conscious of the status of wireless links or the fact that the opponent is on a train.

\subsection{DNS manager}

On-ground name servers, which manage the corre-

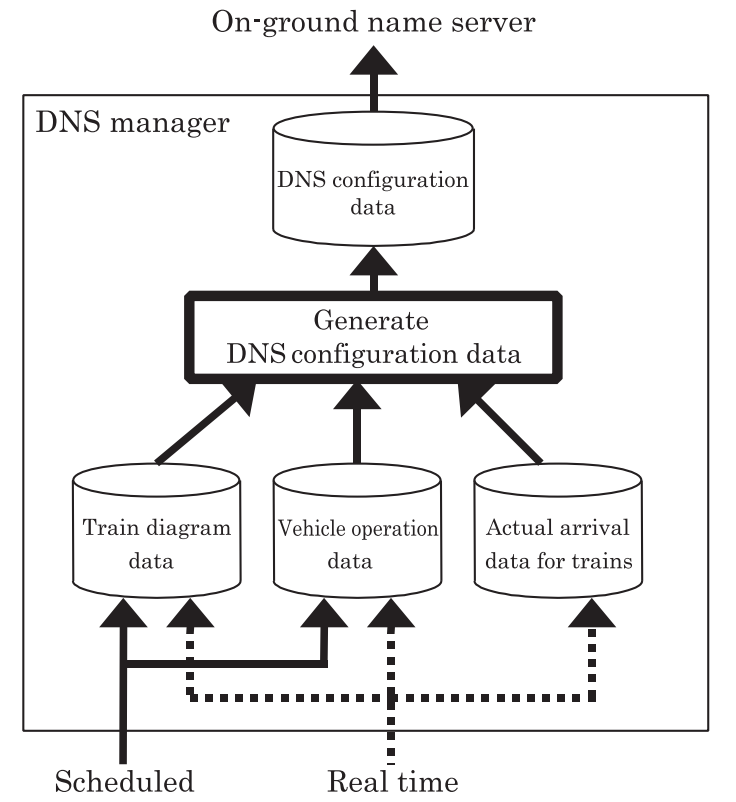

Fig. 8 DNS manager

spondence between IP addresses and host names and provide DNS services, are connected to on-ground networks. DNS manager automatically updates this correspondence according to the status of train operation (Fig. 8). The DNS manager obtains the daily plans of train diagram and vehicle operation when a day starts, and actual arrival data of trains to stations and adjustment schedules on a real-time basis, judges creation or deletion of trains, generates DNS configuration data as a new correspondence, and reflects them on the name servers. A certain time before a train departs from its starting station, for example, the DNS manager generates DNS configuration data which make it possible to refer to the on-board computers of the train with the name containing its train number. 
Table 4 Volume of transferred data

\begin{tabular}{|l|l|l|l|}
\hline & & First time & Second time \\
\hline \hline \multirow{2}{*}{$\begin{array}{l}\text { Customer } \\
\text { information } \\
\text { retrieval }\end{array}$} & Total frame length (kbyte) & 41.6 & 5.9 \\
\cline { 2 - 4 } & Estimated transfer time $(\mathrm{sec})$ & 11.5 & 1.7 \\
\cline { 2 - 4 } & Elapsed time (sec) & 17.5 & 3.2 \\
\hline \multirow{2}{*}{$\begin{array}{l}\text { Travel plan } \\
\text { retrieval }\end{array}$} & Total frame length (byte) & 29.5 & 4.0 \\
\cline { 2 - 4 } & Estimated transfer time $(\mathrm{sec})$ & 8.2 & 1.1 \\
\cline { 2 - 4 } & Total time $(\mathrm{sec})$ & 15.7 & 3.1 \\
\hline
\end{tabular}

\section{Experiments}

\subsection{Transmission characteristics}

We measured the volume of transferred data between the mobile terminal and servers, and evaluated the behavior of the system when the telecommunication environment was unstable.

In these experiments, we used the PHS private mode as the wireless communication channel.

\subsubsection{Volume of transferred data}

We performed a series of information retrieval processes from a mobile terminal twice and measured the total frame length transferred over the PHS channel and the elapsed time between the first frame and the last frame transmitted for each process unit.

Table 4 shows these values and the estimated transfer time which was calculated from the total frame length divided by the transmission rate $(28.8 \mathrm{kbps})$ for the processes of customer detailed information display and travel plan retrieval. As class libraries necessary to perform the process are transmitted from the server at the first time, the total frame is rather long, but it is thought to be allowable for practical use. At the second time or later, only the designated conditions for the retrieval and its results are transmitted, so the total time is very short. The difference between the estimated transfer time and elapsed time is thought to be the database access time.

\subsubsection{Behavior in unstable environment}

Even in the environment where frame errors occur frequently (with about $4 \%$ of the frames damaged) due to very weak radio power, this system was able to operate correctly.

\subsection{Experiment for emulating on-board usage}

We constructed a tentative network in a laboratory to emulate on-board usage shown in Fig. 9. This configuration is for a situation where a train connects to a station network by wireless LAN while it is at a stop at the station. We verified that the system could operate correctly in this environment. As a result, we also verified that the proposed network configuration was valid.

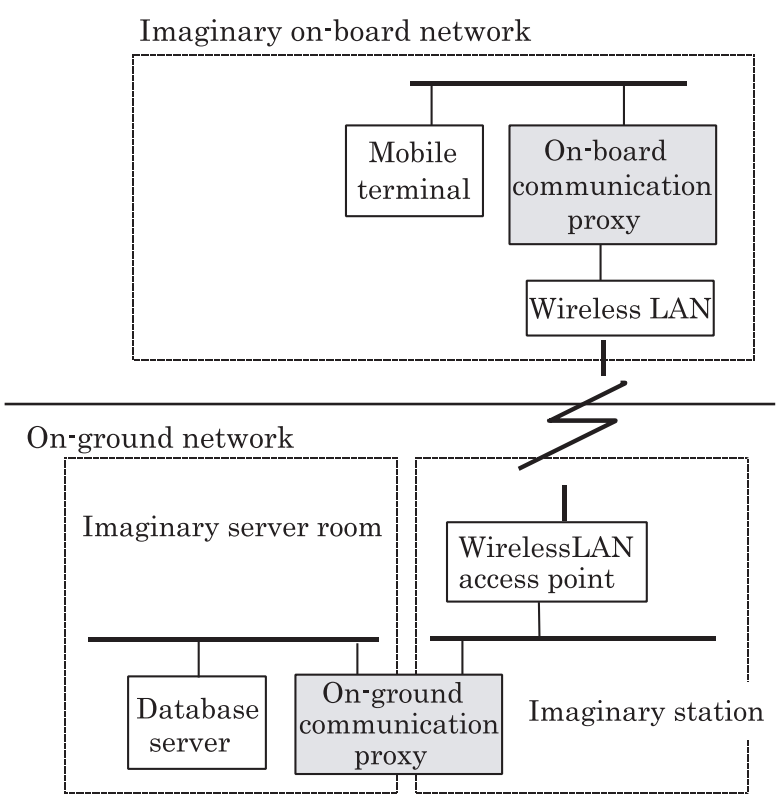

Fig. 9 Configuration of tentative network

\section{Conclusion}

Through the trial manufacture of sales supporting system by the mobile agent technology, we have obtained the prospects of realizing practical systems. We also have showed the possibility of the construction of an Intranet including on-board LAN.

We will continue to study the intelligence of the agent and protocols for the Intranet suitable for mobile objects.

Moreover, we will study adaptation of the system to other applications in addition to the sales supporting system discussed in this paper. By adapting it to the systems which control or monitor on-board devices from the ground (dispatcher or train depot), for example, we can simplify on-board devices by dispatching only an agent which corresponds to the running line or section.

\section{References}

1) Wong,D., Paciorek,N. and Moore,D. :"Java-based Mobile Agents," Communications of the ACM, Vol. 42, No. 3, pp. $92 \sim 102,1999$.

2) Kiniry,J. and Zimmerman,D. :"A hands-on look at Java mobile agents," IEEE Internet Computing, Vol. 1, No. 4, pp. $21 \sim 30,1997$.

3) Lange,D.B. and Oshima,M. :"Programming and Deploying Java ${ }^{\mathrm{TM}}$ Mobile Agents with Aglets ${ }^{\mathrm{TM}}$," Addison-Wesley, 1998.

4) Mockapetris, P. :DOMAIN NAMES - CONCEPTS AND FACILITIES,” RFC 1034, 1987.

5) Mockapetris, P. :"DOMAIN NAMES - IMPLEMENTATION AND SPECIFICATION,” RFC 1035, 1987.

6) Perkins, C., :"IP Mobility Support," RFC 2002, 1998. 